
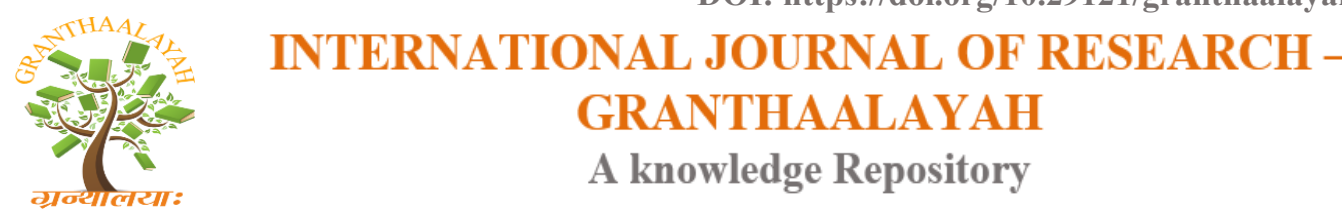

Science

\title{
APPLICATIONS OF EDGE COLORING OF GRAPHS WITH RAINBOW NUMBERS PHENOMENA
}

\author{
Dr. B. Ramireddy ${ }^{1}$, U. Mohan Chand ${ }^{2}$, A. Sri Krishna Chaitanya ${ }^{3}$, Dr. B. R. Srinivas ${ }^{4}$ \\ ${ }^{1}$ Professor \& H.O.D, Hindu College, Guntur, (A.P.), INDIA. \\ ${ }^{2}$ Associate Professor of Mathematics \& H.O.D, Rice Krishna Sai Prakasam Group of \\ Institutions, Ongole, (A.P), INDIA \\ ${ }^{3}$ Associate Professor of Mathematics \& H.O.D, Chebrolu Engineering College, Chebrolu, \\ Guntur Dist. (A.P), INDIA \\ ${ }^{4}$ Associate Professor of Mathematics, St. Mary's Group of Institutions, Chebrolu, Guntur Dist.
}

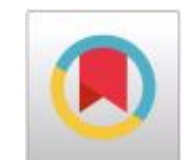
(A.P), INDIA

\section{ABSTRACT}

This paper studies the Rainbow Ramsety Number for a non empty graph and the main results are 1. The Rainbow Ramsety Number of a graph $F$ with out isolated vertices is defined if and only if $F$ is a forest. 2. The Rainbow Ramsety Number of two graphs F1 and F2 with out isolated vertices is defined if and only if $F 1$ is a star or F2 is a forest..

Mathematics Subject Classification 2000: 05CXX, 05C55, 05DXX, 05D10, 04XX, 04A10

Keywords:

Rainbow Ramsety Number, forest, isolated vertices, star.

Cite This Article: Dr. B. Ramireddy, U. Mohan Chand, A. Sri Krishna Chaitanya, and Dr. B. R. Srinivas, "APPLICATIONS OF EDGE COLORING OF GRAPHS WITH RAINBOW NUMBERS PHENOMENA" International Journal of Research - Granthaalayah, Vol. 3, No. 12(2015): 163-170. DOI: https://doi.org/10.29121/granthaalayah.v3.i12.2015.2901.

\section{INTRODUCTION}

Basically in an edge-colored graph $G$ that if there is a sub graph $F$ of $G$ all of whose edges are colored the same, then $\mathrm{F}$ is referred to as a monochromatic F. On the other hand, if all edges of $\mathrm{F}$ are colored differently, then $\mathrm{F}$ is referred to as a rainbow $\mathrm{F}$.

\section{DEFINITION}

For a nonempty graph F, the Rainbow Ramsety Number RR (F) of F as the smallest positive integer $\mathrm{n}$ such that if each edge of the complete graph $\mathrm{K}_{\mathrm{n}}$ is colored from any set of colors, then either a monochromatic $\mathrm{F}$ or a rainbow $\mathrm{F}$ is produced. 
Let $\left\{v_{1}, v_{2}, \ldots . . V_{n}\right\}$ be the vertex set of a complete graph $K_{n}$. An edge coloring of $K_{n}$ using positive integers for colors is called a minimum coloring if two edges $\mathrm{v}_{\mathrm{i}} \mathrm{v}_{\mathrm{j}}$ and $\mathrm{v}_{\mathrm{k}} \mathrm{V}_{\mathrm{l}}$ are colored the same if and only if

$\min \{\mathrm{i}, \mathrm{j}\}=\{\mathrm{k}, \mathrm{l}\}$

while an edge coloring of $K_{n}$ is called a maximum coloring if two edges $u_{i} u_{j}$ and $u_{k} u_{1}$ are colored the same if and only if

$\max \{\mathrm{I}, \mathrm{j}\}=\max \{\mathrm{k}, \mathrm{l}\}$

2.1. Definion: A graph with out cycles is a forest

\subsection{Theorem: Let $\mathbf{F}$ be a graph without isolated vertices. The Rainbow Ramsey number $R R$ ( $F)$ is defined if and only if $F$ is a forest.}

Let $F$ be a graph of order $p \geq 2$. First we show that $R R(F)$ is defined only if $F$ is a forest. Suppose that $F$ is not a forest. Thus $F$ contains a cycle $C$, of length $k \geq 3$ say. Let $n$ be an integer with $n \geq$ $\mathrm{p}$ and let $\left\{\mathrm{v}_{1}, \mathrm{v}_{2}, \ldots \ldots, \mathrm{v}_{\mathrm{n}}\right\}$ be the vertex set of a complete graph $\mathrm{K}_{\mathrm{n}}$. Define an edge coloring $\mathrm{c}$ of $\mathrm{K}_{\mathrm{n}}$ by $\mathrm{c}\left(\mathrm{v}_{\mathrm{i}} \mathrm{v}_{\mathrm{j}}\right)=\mathrm{i}$ if $\mathrm{i}<\mathrm{j}$. Hence $\mathrm{c}$ is a minimum edge coloring of $\mathrm{K}_{\mathrm{n}}$. If $\mathrm{k}$ is the minimum positive integer such that $\mathrm{v}_{\mathrm{k}}$ belongs to $\mathrm{C}$, then two edges of $\mathrm{C}$ are colored $\mathrm{k}$, implying that there is no rainbow $F$ in $K_{n}$. Since any other edge in $C$ is not colored $k$, it follows that $F$ is not monochromatic either. Thus RR (F) is not defined.

For the converse, suppose that $\mathrm{F}$ is a forest of order $\mathrm{p} \geq 2$. By known fact there exists and integer $n \geq p$ such that for any edge coloring of $K_{n}$ with positive integers, there is a complete subgraph $G$ of order $\mathrm{p}$ in $\mathrm{K}_{\mathrm{n}}$ that is either monochromatic or rainbow or has minimum or maximum coloring. If $\mathrm{G}$ is monochromatic or rainbow, then $\mathrm{K}_{\mathrm{n}}$ contains a monochromatic or rainbow $\mathrm{F}$. Hence we may assume that the edge coloring of $\mathrm{G}$ is minimum or maximum, say the former. We show in this case that $\mathrm{G}$ contains a rainbow $\mathrm{F}$. If $\mathrm{F}$ is not a tree, then we can add edges to $\mathrm{F}$ to produce a tree $\mathrm{T}$ of order $\mathrm{p}$. Let

$\mathrm{V}(\mathrm{G})=\left\{\mathrm{v}_{\mathrm{i} 1}, \mathrm{v}_{\mathrm{i} 2}, \ldots \ldots, \mathrm{v}_{\mathrm{ip}}\right)$,

Where $\mathrm{i}_{1}<\mathrm{i}_{2}<\ldots<\mathrm{i}_{\mathrm{p}}$. Select some vertex $\mathrm{v}=\mathrm{v}_{\mathrm{ip}}$ of $\mathrm{T}$ and label the vertices of $\mathrm{T}$ in the order $\mathrm{v}=\mathrm{v}_{\mathrm{ip}}, \mathrm{v}_{\mathrm{ip}-1}, \ldots, \mathrm{v}_{\mathrm{i} 2}, \mathrm{v}_{\mathrm{i} 1}$

of non decreasing distance from $\mathrm{v}$; that is,

$\mathrm{d}\left(\mathrm{v}_{\mathrm{ij}}, \mathrm{v}\right) \geq \mathrm{d}\left(\mathrm{v}_{\mathrm{ij}+1}, \mathrm{v}\right)$

for every integer $j$ with $1 \leq j \leq p-1$. Hence there exists exactly on edge of $T$ having color $i_{j}$ for each $\mathrm{j}$ with $1 \leq \mathrm{j} \leq \mathrm{p}-1$. Thus $\mathrm{T}$ and hence $\mathrm{F}$ is rainbow. The rainbow Ramsey number $R R(F)$ is therefore defined.

\subsection{Example: For each integer $k \geq 2, \operatorname{RR}\left(K_{1, k}\right)=(k-1)^{2}+2$.}

\section{Proof}

We first show that $R R\left(K_{1, k}\right) \geq(k-1)^{2}+2$. Let

$\mathrm{n}=(\mathrm{k}-1)^{2}+1$.

We consider two cases. 
Case 1. $\mathbf{k}$ is odd. Then $n$ is odd Factor $K_{n}$ into ${ }^{n-1} / 2={ }^{(K-1) 2} / 2$ Hamiltonian cycles each. Partition these cycles into $k-1$ sets $S_{i}(1 \leq \mathrm{i} \leq \mathrm{k}-1)$ of ${ }^{\mathrm{k}-1} / 2$ Hamiltonian cycles each. Color each edge of each cycle in $S_{i}$ with color $i$. then there is neither a monochromatic $K_{1, k}$ nor a rainbow $K_{1, k}$.

Case 2. $k$ is even. Then $n$ is even. Factor $K_{n}$ into $n-1=(k-1)^{2} 1$-factors. Partition these 1 -factors into $k-1$ sets $S_{i}(1 \leq \mathrm{i} \leq \mathrm{k}-1)$ of $\mathrm{k}-1$-factors. Color each edge of each 1 -factor in $S_{\mathrm{i}}$ color with $\mathrm{i}$. Then there is neither a monochromatic $\mathrm{K}_{1, \mathrm{k}}$ nor a rainbow $\mathrm{K}_{1, \mathrm{k}}$.

Therefore, $\mathrm{RR}\left(\mathrm{K}_{1, \mathrm{k}}\right) \geq(\mathrm{k}-1)^{2}+2$. It remains to show that $\mathrm{RR}\left(\mathrm{K}_{1, \mathrm{k}}\right) \leq(\mathrm{k}-1)^{2}+2$. Let $\mathrm{N}=(\mathrm{k}-$ $1)^{2}+2$ and let there be given an edge coloring of $K_{N}$ from any set of colors. Suppose that no monochromatic $\mathrm{K}_{1, \mathrm{k}}$ results. Let $\mathrm{v}$ be a vertex of $\mathrm{K}_{\mathrm{N}}$. Since $\operatorname{deg} \mathrm{v}=\mathrm{N}-1$ and there is no monochromatic $\mathrm{K}_{1, \mathrm{k}}$, at most $\mathrm{k}-1$ edges incident with $\mathrm{v}$ can be colored the same. Thus there are at least $\left[{ }^{\mathrm{N}} / \mathrm{k}-1\right]=\mathrm{k}$ edges incident with $\mathrm{v}$ that are colored differently, producing a rainbow $\mathrm{K}_{1, \mathrm{k}}$.

More generally, for two nonempty graphs $F_{1}$ and $F_{2}$, the rainbow Ramsey number $R R\left(F_{1}, F_{2}\right)$ is defined as the smallest positive integer $n$ such that if each edge of $K_{n}$ is colored from any set of colors, then there is either a monochromatic $F_{1}$ or a rainbow $F_{2}$ defined for every pair $F_{1}, F_{2}$ of non empty graphs.

\section{DEFINITION}

If the partite sets $\mathrm{u} \& \mathrm{w}$ of a complete bi partite graph contain s\&t vertices. Then this graph is denoted by $\mathrm{K}_{\mathrm{s}, \mathrm{t}}$.the graph $\mathrm{K}_{1, \mathrm{t}}$ is called star.

\subsection{Theorem: Let $F_{1}$ and $F_{2}$ be two graphs without isolated vertices. The rainbow Ramsey number $R R\left(F_{1}, F_{2}\right)$ is defined if and only if $F_{1}$ is a star or $F_{2}$ is a forest.}

Proof. First, we show that $R R\left(F_{1}, F_{2}\right)$ is exists only if $F_{1}$ is a star or $F_{2}$ is a forest. Suppose that $F_{1}$ is not a star and $F_{2}$ is not a forest. Let $G$ be a complete graph of some order $n$ such that $V(G)=$ $\left\{\mathrm{v}_{1}, \mathrm{v}_{2}, \ldots, \mathrm{v}_{\mathrm{n}}\right\}$ and such that both $\mathrm{F}_{1}$ and $\mathrm{F}_{2}$ are sub graphs of $\mathrm{G}$. Define an $(\mathrm{n}-1)$-edge coloring on $\mathrm{G}$ such that the edge $\mathrm{v}_{\mathrm{i}} \mathrm{v}_{\mathrm{j}}$ is assigned the color $\mathrm{i}$ if $\mathrm{i}<\mathrm{j}$. Hence this coloring is a minimum edge coloring of $\mathrm{G}$.

Let $G_{1}$ be any copy of $F_{1}$ in $G$ and let a be the minimum integer such that $v_{a}$ is a vertex of $G_{1}$. Then every edge incident with $v_{a}$ is colored a. since $G_{1}$ is not a star, some edge of $G_{1}$ is not incident with $\mathrm{v}_{\mathrm{a}}$ and is therefore not colored a. Hence $\mathrm{G}_{1}$ is not monochromatic. Next, let $\mathrm{G}_{2}$ be any copy of $F_{2}$ in $G$. Since $G_{2}$ is not a forest, $G_{2}$ contains a cycle $C$. Let $b$ be the minimum integer such that $v_{b}$ is a vertex of $G_{2}$ belonging to $C$. Since the two edges of $C$ incident with $v_{b}$ are colored $b$ (and $G_{2}$ contains at least two edges colored $b$ ), $G_{2}$ is not a rainbow subgraph of $G$. Hence $\mathrm{RR}\left(\mathrm{F}_{1}, \mathrm{~F}_{2}\right)$ is not defined.

We now verify the converse. Let $F_{1}$ and $F_{2}$ be two graphs without isolated vertices such that either $F_{1}$ is a star or $F_{2}$ is a forest. We show that there exists a positive integer $n$ such that for every edge coloring of $\mathrm{K}_{\mathrm{n}}$, either a monochromatic $\mathrm{F}_{1}$ or a rainbow $\mathrm{F}_{2}$ results. Suppose that the order of $F_{1}$ is $s+1$ and the order of $F_{2}$ is 
$t+1$ for positive integers $s$ and $t$. Hence $F_{1}=K_{1, s}$. We now consider two cases, depending on whether $F_{1}$ is a star or $F_{2}$ is a forest. It is convenient to begin with the case where $F_{2}$ is a forest.

Case 1. $F_{2}$ is a forest. If $F_{2}$ is not a tree, then we may add edges to $F_{2}$ so that a tree $G_{2}$ results. If $F_{2}$ is a tree, then let $G_{2}=F_{2}$. Furthermore, if $F_{1}$ is not complete, then we may add edges to $F_{1}$ so that a complete graph $\mathrm{G}_{1}=\mathrm{K}_{\mathrm{s}+1}$ results. If $\mathrm{F}_{1}$ is complete, then let $\mathrm{G}_{1}=\mathrm{F}_{1}$. Hence $\mathrm{G}_{1}=\mathrm{K}_{\mathrm{s}+1}$ and $\mathrm{G}_{2}$ is a tree of order $t+1$. We now show that $R R\left(G_{1}, G_{2}\right)$ is defined by establishing the existence of a positive integer $n$ such that any edge coloring of $\mathrm{K}_{n}$ from any set of colors results in either a monochromatic $G_{1}$ or a rainbow $G_{2}$. This, in turn, implies the existence of monochromatic $F_{1}$ or a rainbow $F_{2}$. We now consider two sub cases, depending on whether $G_{2}$ is a star.

Sub case 1.1. $G_{2}$ is a star of order $t+1$, that is, $G_{2}=K_{1, t}$. Therefore, in this subcase, $G_{1}=K_{s+1}$ and $G_{2}=K_{1, t}$ (This subcase will aid us later in the project) In this subcase, let

$$
n=\sum_{i=0}^{(s-1)(t-1)+1}(t-1)^{i}
$$

and let an edge coloring of $\mathrm{K}_{\mathrm{n}}$ be given from any set of colors. If $\mathrm{K}_{\mathrm{n}}$ contains a vertex incident with $t$ or more edges assigned distinct colors, then $K_{n}$ contains a rainbow $G_{2}$. Hence we may assume that every vertex of $K_{n}$ is incident with at most $t-1$ edges assigned distinct colors. Let $v_{1}$ be a vertex of $K_{n}$. Since the degree of $v_{1}$ in $K_{n}$ is $n-1$, there are at least.

$$
\frac{n-1}{t-1}=\sum_{i=0}^{(s-1)(t-1)}(t-1)^{i}
$$

edges incident with $\mathrm{u}_{1}$ that are assigned the same color, say color $\mathrm{c}_{1}$.

Let $S_{1}$ be the set of vertices joined to $v_{1}$ by edges colored $c_{1}$ and let $v_{2} \in S_{1}$.

There are at least

$$
\frac{\left|S_{1}\right|-1}{t-1} \geq \sum_{i=0}^{(s-1)(t-1)-1}(t-1)^{i}
$$

edges of the same color, say color $c_{2}$, joining $v_{2}$ and vertices of $S_{1}$, where possibly $c_{2}=c_{1}$. Let $S_{2}$ be the set of vertices in $S_{1}$ joined to $v_{2}$ by edges colored $c_{2}$. Continuing in this manner, we construct sets $\mathrm{S}_{1}, \mathrm{~S}_{2}, \ldots . . \mathrm{S}_{(\mathrm{s}-1)(\mathrm{t}-1)}$ and vertices, $\mathrm{v}_{1}, \mathrm{~V}_{2}, \ldots . \mathrm{v}_{(\mathrm{s}-1)(\mathrm{t}-1)+1}$ such that $2 \leq \mathrm{i} \leq(\mathrm{s}-1)(\mathrm{t}-1)+1$, the vertex $v_{i}$ belongs to $S_{i-1}$ and is joined to at least

$$
\frac{\left|S_{1}\right|-1}{t-1} \geq \sum_{i=0}^{(s-1)(t-1)-1}(t-1)^{i}
$$

vertices of $S_{i-1}$ by edges colored $c_{i}$. Finally, in the set $S_{(s-1)(t-1)}$, the vertex 
$\mathrm{v}_{(\mathrm{s}-1)(\mathrm{t}-1)+1}$ is joined to a vertex $\mathrm{v}_{(\mathrm{s}-1)(\mathrm{t}-1)+2}$ in $\mathrm{S}_{(\mathrm{s}-1)(\mathrm{t}-1)}$ by an edge colored $\mathrm{c}_{(\mathrm{s}-1)(\mathrm{t}-1)+1}$. Thus we have a sequence

$$
v_{1}, v_{2}, \ldots, v_{(s-1)}(t-1)+2
$$

of vertices such that every edge $\mathrm{v}_{\mathrm{i}} \mathrm{v}_{\mathrm{j}}$ for $1 \leq \mathrm{i}<\mathrm{j} \leq(\mathrm{s}-1)(\mathrm{t}-1)+2$ is colored $\mathrm{c}_{\mathrm{i}}$ and where the colors $\mathrm{c}_{1}, \mathrm{c}_{2}, \ldots . \mathrm{c}_{(\mathrm{s}-1)(\mathrm{t}-1)+1}$ are not necessarily distinct. In the complete subgraph $\mathrm{H}$ of order $(\mathrm{s}-1)$ $(\mathrm{t}-1)+2$ induced by the vertices listed in (11.3), the vertex $\mathrm{v}(\mathrm{s}-1)(\mathrm{t}-1)+2$ is incident with at most $\mathrm{t}$ -1 edges having distinct colors. Hence there is a set of at least.

$$
\left\lceil\frac{(s-1)(t-1)+1}{t-1}\right\rceil=s
$$

Vertices in $\mathrm{H}$ joined to $\mathrm{V}(\mathrm{s}-1)(\mathrm{t}-1)+2$ by edges of the same color. Let $\mathrm{V}_{\mathrm{i} 1}, \mathrm{~V}_{\mathrm{i} 2}, \ldots, \mathrm{V}_{\mathrm{is}}$ be $\mathrm{s}$ of these vertices, where $\mathrm{i}_{1}<\mathrm{i}_{2}<\ldots \ldots<\mathrm{i}_{\mathrm{s}}$. Then $\mathrm{c}_{\mathrm{i} 1}=\mathrm{c}_{\mathrm{i} 2}=\ldots . .=\mathrm{c}_{\mathrm{is}}$, and the complete subgraph of order $\mathrm{s}+$ 1 induced by

$\left\{\mathrm{v}_{\mathrm{i} 1}, \mathrm{v}_{\mathrm{i} 2}, \ldots, \mathrm{v}_{\mathrm{is}}, \mathrm{v}_{(\mathrm{s}-1)(\mathrm{t}-1)+2}\right\}$

is monochromatic.

Subcase $1.2 G_{2}$ is a tree of order $t+1$ that is not necessarily a star. Recall that $G_{1}=K_{s+1}$. We proceed by induction on the positive integer $t$. If $t=1$ or $t=2$, then $G_{2}$ is a star and the base case of the induction follows by subcase 1.1. Suppose that $R R\left(G_{1}, G_{2}\right)$ exists for $G_{1}=K_{s+1}$ and for every tree $G_{2}$ of order $t+1$ where $t \geq 2$. Let $T$ be a tree of order $t+2$. We show that $R R\left(G_{1}, T\right)$ exists. Let $\mathrm{v}$ be an end-vertex of $\mathrm{T}$ and let $\mathrm{v}$ be the vertex of $\mathrm{T}$ that is adjacent to $\mathrm{v}$. Let $\mathrm{T}^{\mathrm{l}}=\mathrm{T}-$ v. Since $T^{l}$ is a tree of order $t+1$, it follows by the induction hypothesis that $R R\left(G_{1}, T^{l}\right)$ exists, say $R R\left(G_{1}, T^{l}\right)=p$. Hence for any edge coloring of $K_{p}$ from any set of colors, there is either a monochromatic $G_{1}=K_{s+1}$ or a rainbow $T^{1}$. From sub case 1.1 , we know that $R R\left(G_{1}, K_{1, t+1}\right)$ exists. Suppose that $R R\left(\mathrm{G}_{1}, \mathrm{~K}_{1, \mathrm{t}+1}\right)=\mathrm{q}$ and let $\mathrm{n}=\mathrm{pq}$ in this subcase.

Let there be given an edge coloring of $\mathrm{K}_{\mathrm{n}}$ using any number of colors. Consider a partition of the vertex set of $K_{n}$ into $q$ mutually disjoint sets of $p$ vertices each. By the induction hypothesis, the complete subgraph induce by each set of $\mathrm{p}$ vertices contains either a monochromatic $\mathrm{K}_{\mathrm{s}+1}$ or rainbow $\mathrm{T}^{1}$. If a monochromatic $\mathrm{K}_{\mathrm{s}+1}$ occurs in any of these complete subgraph $\mathrm{K}_{\mathrm{p}}$, then subcase 1.2 is verified. Hence we may assume that there are q pair wise mutually rainbow copies.

$\mathrm{T}_{1}^{1}, \mathrm{~T}_{2}^{1}, \ldots, \mathrm{T}_{\mathrm{q}}^{1}$

of $\mathrm{T}^{1}$, where $\mathrm{u}_{\mathrm{i}}$ is the vertex in $\mathrm{T}_{\mathrm{i}}^{1}(1 \leq \mathrm{i} \leq \mathrm{q})$ corresponding to the vertex $\mathrm{u}$ in $\mathrm{T}^{1}$.

Let $\mathrm{H}$ be the complete subgraph of order $\mathrm{q}$ induced by $\left\{\mathrm{u}_{1}, \mathrm{u}_{2}, \ldots, \mathrm{u}_{\mathrm{q}}\right\}$. Since $\mathrm{RR}\left(\mathrm{K}_{\mathrm{s}+1}, \mathrm{~K}_{1, t+1}\right)=\mathrm{q}$, it follows that either $\mathrm{H}$ contains a monochromatic $\mathrm{K}_{\mathrm{s}+1}$ or a rainbow $\mathrm{K}_{1, t+1}$. If $\mathrm{H}$ contains a monochromatic $\mathrm{K}_{\mathrm{s}+1}$, then once again, the proof of subcase 1.2 is complete. So we may assume that $\mathrm{H}$ contains a rainbow $\mathrm{K}_{1, t+1}$. Let $\mathrm{u}_{\mathrm{j}}$ be the center of a rainbow star $\mathrm{K}_{1, t+1}$ in $\mathrm{H}$. At least one of the $t+1$ colors of the edges of $K_{1, t+1}$ is different from the colors of the $t$ edges of $T_{j}^{1}$ Adding the edge having this color at $u_{j}$ in $T_{j}^{1}$ produces a rainbow copy of $T$. 
Case 2. $F_{1}$ is a star. Denote $F_{1}$ by $G_{1}$ as well and so $G_{1}=K_{1, \text { s. If }} F_{2}$ is complete, then let $G_{2}=F_{2}$. If $F_{2}$ is not complete, then we may add edges to $F_{2}$ so that a complete graph $G_{2}=K_{t+1}$ results. We verify that $R R\left(G_{1}, G_{2}\right)$ exists by establishing the existence of a positive integer $n$ such that for any edge coloring of $K_{n}$ from any set of colors, either a monochromatic $G_{1}$ or a rainbow $G_{2}$ results. This then shows that $K_{n}$ will have a monochromatic $F_{1}$ or a rainbow $F_{2}$. For positive integers $\mathrm{p}$ and $\mathrm{r}$ with $\mathrm{r}<\mathrm{p}$, let

$$
p^{(r)}=\frac{p !}{(p-r) !}=p(p-1) \cdots(p-r+1)
$$

Now let $\mathrm{n}$ be an integer such that $\mathrm{s}-1$ divides $\mathrm{n}-1$ and

$$
n \geq 3+\frac{(s-1)(t+2)^{(4)}}{8}
$$

Then $\mathrm{n}-1=(\mathrm{s}-1) \mathrm{q}$ for some positive integer $\mathrm{q}$. Let there be given an edge coloring of $\mathrm{K}_{\mathrm{n}}$ from any set of colors and suppose that no monochromatic $G_{1}=K_{1, s}$ occurs. We show that there is a

rainbow $\mathrm{G}_{2}=\mathrm{K}_{\mathrm{t}+1}$. Observe that the total number of different copies of $\mathrm{K}_{\mathrm{t}+1}$ in $\mathrm{K}_{\mathrm{n}}$ is $\left(\begin{array}{c}n \\ t+1\end{array}\right)$ implying the existence of at least one rainbow $\mathrm{K}_{\mathrm{t}+1}$.

First consider the number of copies of $\mathrm{K}_{\mathrm{t}+1}$ containing adjacent edges $\mathrm{uv}$ and uw that are colored the same. There are $n$ possible choice for the vertex $u$. suppose that there are $a_{i}$ edges incident with $\mathrm{u}$ that are colored $\mathrm{i}$ for $1 \leq \mathrm{i} \leq \mathrm{k}$. Then

$$
\sum_{i=1}^{k} a_{i}=n-1
$$

Where, by assumption, $1 \leq \mathrm{a}_{\mathrm{i}} \leq \mathrm{s}-1$ for each $\mathrm{i}$. For each color $\mathrm{i}(1 \leq \mathrm{i} \leq \mathrm{k})$, the number of different choices for $\mathrm{v}$ and $\mathrm{w}$ where $\mathrm{uv}$ and $\mathrm{uw}$ are colored $\mathrm{i}$ is $\left(\begin{array}{c}a_{i} \\ 2\end{array}\right)$. Hence the number of different choices for $\mathrm{u}$ and $\mathrm{w}$ where $u v$ and $u w$ are colored the same is

$$
\sum_{i=1}^{k}\left(\begin{array}{c}
a_{i} \\
2
\end{array}\right)
$$

since the maximum value of this sum occurs when each $a_{i}$ is as large as possible, the largest value of this sum is when each $a_{i}$ is $s-1$ and when $k=q$, that is, there are at most

$$
\sum_{i=1}^{q}\left(\begin{array}{c}
s-1 \\
2
\end{array}\right)=q\left(\begin{array}{c}
s-1 \\
2
\end{array}\right)
$$


choices for $\mathrm{v}$ and $\mathrm{w}$ such that $\mathrm{uv}$ and uw are colored the same. Since there are $\left(\begin{array}{c}n-3 \\ t-2\end{array}\right)$ choices for the remaining $\mathrm{t}-2$ vertices of $\mathrm{K}_{\mathrm{t}+1}$, it follows that there are at most

$$
n q\left(\begin{array}{c}
s-1 \\
2
\end{array}\right)\left(\begin{array}{c}
n-3 \\
t-2
\end{array}\right)
$$

copies of $\mathrm{K}_{\mathrm{t}+1}$ containing two adjacent edges that are colored the same.

We now consider copies of $K_{t+1}$ in which there two nonadjacent edges, say e = xy and $f=w z$,

$$
\left(\frac{n}{2}\right)
$$

choices for e and $n-2$ choices for one vertex, say $w$, that is incident with $\mathrm{f}$. The vertex $\mathrm{w}$ is incident with at most $\mathrm{s}-1$ edges having the same color as e and not adjacent to e. Since there are four ways of counting such a pair of edges in this way (namely e and either $\mathrm{w}$ or $\mathrm{z}$, or $\mathrm{f}$ and either $\mathrm{x}$ or $\mathrm{y})$, there are at most

$$
\frac{\left(\begin{array}{l}
n \\
2
\end{array}\right)(n-2)(s-1)}{4}=\frac{n(n-1)(n-2)(s-1)}{8}
$$

Ways to choose nonadjacent edges of the same color and $\left(\begin{array}{l}n-4 \\ t-3\end{array}\right)$ ways to choose the remaining $\mathbf{t}$ 3 vertices of $\mathrm{K}_{\mathrm{t}+1}$. Hence there are at most.

$$
\frac{n(n-1)(n-2)(s-1)}{8}\left(\begin{array}{c}
n-4 \\
t-3
\end{array}\right)
$$

Copies of $\mathrm{K}_{\mathrm{t}+1}$ containing two nonadjacent edges that are colored the same. Therefore, the number of non-rainbow copies of $\mathrm{K}_{\mathrm{t}+1}$ is at most

$$
\begin{aligned}
& n q\left(\begin{array}{c}
s-1 \\
2
\end{array}\right)\left(\begin{array}{c}
n-3 \\
t-2
\end{array}\right)+\frac{n(n-1)(n-2)(s-1)}{8}\left(\begin{array}{c}
n-4 \\
t-3
\end{array}\right) \\
= & n\left(\begin{array}{l}
n-1 \\
s-1
\end{array}\right) \frac{(s-1)(s-2)}{2}\left(\frac{n-2}{n-2}\right)\left(\begin{array}{c}
n-3 \\
t-2
\end{array}\right) \\
& \quad+\frac{n(n-1)(n-2)(s-1)}{8}\left(\frac{n-3}{n-3}\right)\left(\begin{array}{c}
n-4 \\
t-3
\end{array}\right) \\
= & \left(\begin{array}{c}
n \\
t+1
\end{array}\right)\left[\frac{(s-2)(t+1)^{(3)}}{2(n-2)}+\frac{(s-1)(t+1)^{(4)}}{8(n-3)}\right] \\
< & \left(\begin{array}{c}
n \\
t+1
\end{array}\right)\left[\frac{(s-1)(t+1)^{(3)}}{2(n-3)}+\frac{(s-1)(t+1)^{(4)}}{8(n-3)}\right] \\
= & \left(\begin{array}{c}
n \\
t+1
\end{array}\right)\left[\frac{(s-1)(t+1)^{(3)}(t+2)}{8(n-3)}\right] \\
= & \left(\begin{array}{c}
n \\
t+1
\end{array}\right)\left[\frac{(s-1)(t+2)^{(4)}}{8(n-3)}\right] \leq\left(\begin{array}{c}
n \\
t+1
\end{array}\right),
\end{aligned}
$$


Where the final inequality follows from known theorem, the rainbow Ramsey number is defined if and only if $\mathrm{F}$ is a forest hence there is a rainbow $\mathrm{K}_{\mathrm{t}+1}$ in $\mathrm{K}_{\mathrm{n}}$.

\section{REFERENCES}

[1] B.Bollobas \& A.J.Harris, list colorings of graphs. Graphs combin.1(1985) 115-127

[2] G.Chartrand, G.L.Johns, K.A.McKeon, and P.Zhang, Rainbow Connection in Graphs. Math. Bohem.

[3] G.Chartrand, G.L.Johns, K.A.McKeon, and P.Zhang, Rainbow Connectivity of a Graphs. Networks.

[4] C.A.Christen and S.M.Selkow, some perfect coloring properties of Graphs.

[5] J.Combin.Theory Ser. B27 (1979) 49-59.

[6] S.Fiorini \& R.J.Wilson, edge colorings of graphs.Pitman,London (1977). 